\title{
Heat Technology Aiming to Mitigate Structural Heat Sinks for Drywood Termite (Blattodea: Kalotermitidae) Management
}

\author{
Jia-Wei Tay ${ }^{1 *}$, Devon James ${ }^{2}$
}

1 Urban Entomology Laboratory, Department of Plant and Environmental Protection Sciences, University of Hawaii at Manoa, 3050 Maile Way, Gilmore Hall 310, Honolulu, HI 96822; jwtay@hawaii.edu

2 Hi-Temp Tech, LLC., 2877 Kalakaua Ave, Honolulu, HI 96815; info@hitemp.tech

* Correspondence: jwtay@hawaii.edu

\begin{abstract}
Simple Summary: The West Indian drywood termite, Cryptotermes brevis poses a significant economic threat in Hawaii, the southeast portion of the continental United States, and throughout tropical and subtropical regions worldwide. Heat treatment is the only nonchemical option to manage them. A typical heat treatment may not be able to provide a complete kill of drywood termites due to the presence of difficult-to-heat areas in structures. To mitigate this effect, studies were conducted in drywood termite-infested homes in Honolulu, Hawaii, where either a standard heat treatment performed by a heat remediation company or improved heat treatment methods were used. For improved treatments, heated air was directed into the drilled difficult-to-heat areas to distribute heat evenly. Eight temperature sensors were placed in the treatment zone to ensure sufficiently high heat reached all areas. Target temperatures above $46^{\circ} \mathrm{C}$ or $50{ }^{\circ} \mathrm{C}$ that would kill termites were recorded in all areas throughout the 120-minute treatment period. A pretreatment inspection was conducted, and follow-up inspections were performed at 6 months posttreatment to confirm termite inactivity using visual observations and a termite detection device. In improved treatment homes, no termite activity was found after treatment. Guidelines for the improved heat treatment are proposed.
\end{abstract}

\begin{abstract}
With heat treatments to control drywood termites (Blattodea: Kalotermitidae), the presence of heat sinks, which have insulating properties, causes heat to be distributed unevenly throughout the treatment areas. Drywood termites may move to galleries in heat sink areas to avoid exposure to lethal temperatures. To mitigate heat sink effect, studies were conducted in Crytotermes brevis-infested homes in Honolulu, Hawaii to reflect real-world scenarios; either a standard heat treatment performed by a heat remediation company or improved heat treatment methods were used. For improved treatments, heated air was directed into the toe-kick voids of cabinets to reduce heat sink effects. Eight thermistor sensors were placed inside toe-kick voids, in the treatment zone, embedded inside cabinets or the sidewall, or in a wooden cube to monitor internal and ambient temperatures to ensure sufficiently high heat reached all areas. Target temperatures above $46{ }^{\circ} \mathrm{C}$ or $50{ }^{\circ} \mathrm{C}$ were recorded in all areas for 120 minutes. A pretreatment inspection was conducted, and follow-up inspections were performed at 6 months posttreatment to confirm termite inactivity using visual observations and a Termatrac device. In improved treatment homes, no termite activity was found after treatment. Efficacious heat treatment protocols for structures using the improved method are proposed.
\end{abstract}

Keywords: heat treatment; termite control; termites; drywood termite; Crytotermes brevis; wood pest; heat sink; heat technology; non-chemical; pest management

\section{Introduction}


Major drywood termite species found in Hawaii include Cryptotermes brevis, Incisitermes immigrans, and Neotermes connexus [1-3]. Among these species, the West Indian drywood termite, C. brevis, is the most damaging and prevalent in structures and has been recorded in Hawaii since 1884 [4]. This termite poses a significant economic threat in Hawaii and the southeast portion of the continental United States as well as throughout tropical and subtropical regions worldwide [5-11].

Wood-inhabiting insects such as drywood termites can be easily spread in infested wood products through transportation and commerce [12]. Evidence of drywood termite infestation is often the presence of fecal pellets, more commonly known as "frass," which are usually ejected from "kick-out holes" excavated by termites in the wood surface [13]. Their cryptic nature and slow colony growth make drywood termite infestations difficult to detect until colonies are well established.

Heat is an effective means of control for pests infesting structures and commodities [14] and has been used against drywood termites, bedbugs, stored product pests, and powderpost beetles [15-18]. In general, two types of heat sources are used for heat treatment within structures: propane and electricity. Both heat source types generate and release heated air into a structure, usually with the aid of several fans to circulate the hot air throughout the target areas. During the heat treatment, temperature sensors or thermocouples are used to monitor the ambient or internal temperatures of the treatment areas. The treatment area is covered with thermal blankets or tarpaulins to contain the heated air and subsequently improve the heating efficiency. The treatment process involves heating the immediate environment of the target pest to a lethal temperature $\left(\sim 49^{\circ} \mathrm{C}\right)$. Depending on several factors (e.g., the degree of infestation, structural materials, size and configuration of the treatment area, and ambient external temperature), treatment time will take from 1-2 hours up to a few days to reach and maintain the target temperatures for an adequate period.

Ebeling (1975) [19] first documented the use of high temperature of $49{ }^{\circ} \mathrm{C}$ for drywood termite management. In laboratory studies, Woodrow and Grace (1998a) [20] found that $51.3^{\circ} \mathrm{C}$ was lethal to C. brevis, and that a wood-core temperature of $54.4{ }^{\circ} \mathrm{C}$ was sufficient to kill C. brevis in large timbers [21]. Scheffrahn et al. (1997b) [22] also reported that complete mortality was observed in the pseudergates of drywood termite species including C. brevis when the internal wood temperature was maintained at $54.4^{\circ} \mathrm{C}$ for one hour.

One of the challenges in performing an efficacious heat treatment is the presence of structural heat sinks or difficult-to-heat areas [23,24]. These areas may be found in concrete, tile, or insulating materials contacting the target wood members. A toe-kick, sometimes known as a toe space, is the recessed area between the bottom of a cabinet and the floor. This is a common design in kitchens and bathrooms and is a potential heat sink zone. Heat sinks require higher temperatures than other areas to heat the wood sufficiently to kill termites. Furthermore, a field study Woodrow and Grace (1998c) [25] conducted showed that the temperature may increase more slowly in field applications than in laboratory studies. Depending on the degree of wood surface area exposed to heated air, a higher temperature or longer heating time may be needed to achieve the optimum internal temperature in the field.

This heat sink factor does not appear to have been thoroughly considered in developing protocols for heat treatment for drywood termite control in actual homes or condominium units. Our research investigated the efficacy of heat treatment with the aim of eliminating or reducing heat sinks by using a novel method to direct the heated air into the toe-kick voids in homes infested with C. brevis. Using this information, guidelines for more efficacious heat treatment are proposed. 


\section{Materials and Methods}

Study site and protocols

The study took place in two occupied, fully furnished residential condominium units in Honolulu, Hawaii, built between 2001 and 2006. Honolulu is located on the island of Oahu, Hawaii. This region is defined as tropical, with an average annual temperature of $20.6-27.5^{\circ} \mathrm{C}$, the ideal temperature range for termite activity. The homeowners had noted fecal pellets in their cabinets. Upon visual inspection, C. brevis pseudergates and a soldier were found inside a few bases of the cabinets and inside the plywood countertop underlayment, and termite damage or infestation signs were found at those locations (Figure 1). In each home, either a termite soldier or a dead soldier's head was collected in vials with $70 \%$ ethanol and was identified in the laboratory according to an identification guide [7]. Using a low-energy microwave termite detection device commercially known as Termatrac (T3i All Sensor, Termatrac, CA, USA), three 10-minute inspections were carried out to confirm termite activity and the locations of infestation in each home. The device was mounted on a tripod to minimize shaking, and all residents were absent during the inspections to reduce movement around the device. Careful observations of fluctuations in the output signal were conducted. All factors that may generate false-positive results on Termatrac were considered following Taravati (2018) [26]. Heat treatments were subsequently conducted only in those parts of the condominium units (e.g., kitchens) with apparent termite activity. HI-Temp Tech, LLC., a Hawaiibased company that provides thermal remediation services, conducted the heat treatments.

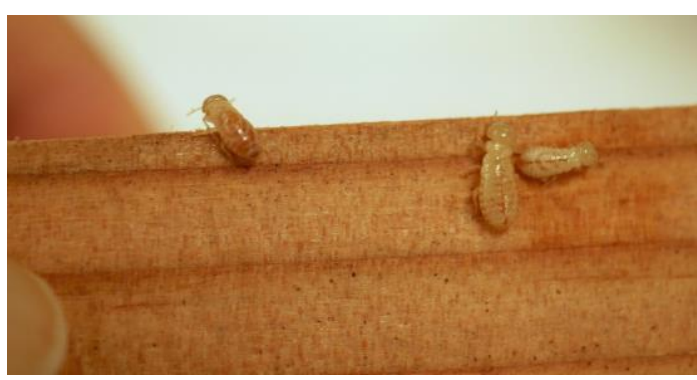

(A)

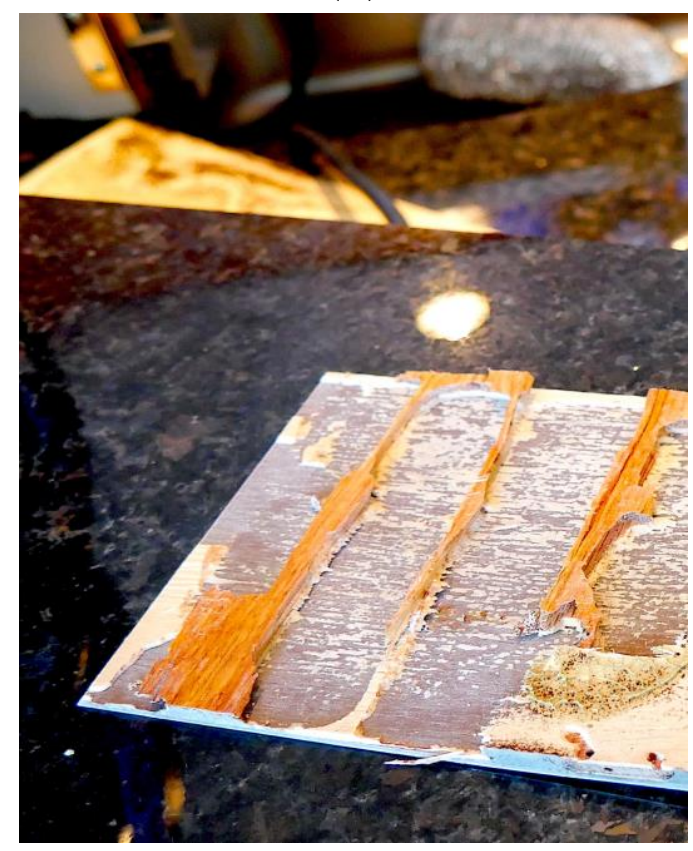

(B) 
Figure 1. (A,B) A few drywood termite pseudergates nested inside damaged wood were separated and transferred to another piece of undamaged wood and were kept in a transparent plastic box to facilitate general identification and photography (A). Termite infestation signs at parts of condominium units: Termite damage and some unrelated water damage noted on cabinet woods (B).

Before performing the treatments, furniture and personal items were moved away from infested areas to allow access for heat application. All electrical appliances (e.g., microwaves, kettles, and toasters) were removed to increase air circulation and heat penetration. Additionally, all air conditioners and air vents were covered and sealed with tape, and sink drains were taped to avoid P-trap evaporation. Fire sprinklers were insulated as necessary. These steps improve heating efficiency, help hermetically seal the treatment zone, and reflect heat energy into the treatment areas. All cabinet doors and drawers were in open and staggered positions.

Because the $C$. brevis infestations at these homes were associated with cabinets, the base cabinets with toe-kick voids were identified as the areas most prone to slow rates of temperature increases. For improved treatment homes, a four-inch-diameter hole was drilled in the back of each infested base cabinet with toe-kick voids (Figure 2). For each home, a total of three thermistor probes were placed deep inside the toe-kick voids next to the largest or deepest sidewall or baseboard to monitor and record temperatures to ensure that the heat thoroughly reached all areas, including these heat sink areas (Figure 3). Two ambient air temperature sensors were placed at recessed corners of the treatment zones. Two core temperature probes were installed by drilling to the center of the selected sidewall or side cabinet, placing them in the hole, and sealing the hole with duct tape (Figure 4a). Additionally, one custom-made wooden cube with an embedded sensor in layered woods, which simulated the thickness of the cabinet wood but may have been thicker than the actual cabinet wood to represent potential worst case areas of different home conditions, was placed in a low-circulation location for temperature recording (Figure $4 \mathrm{~b}$ ). Temperatures from a total of eight wireless sensors were monitored from an online wireless portal (iMonnit, AZ, USA) in each treated home. A cellular gateway and control unit were set up in each treatment home to collect the thermistor probe and sensor readings and upload sensor data in batches at 10-minute intervals to the online portal (Figure 5).
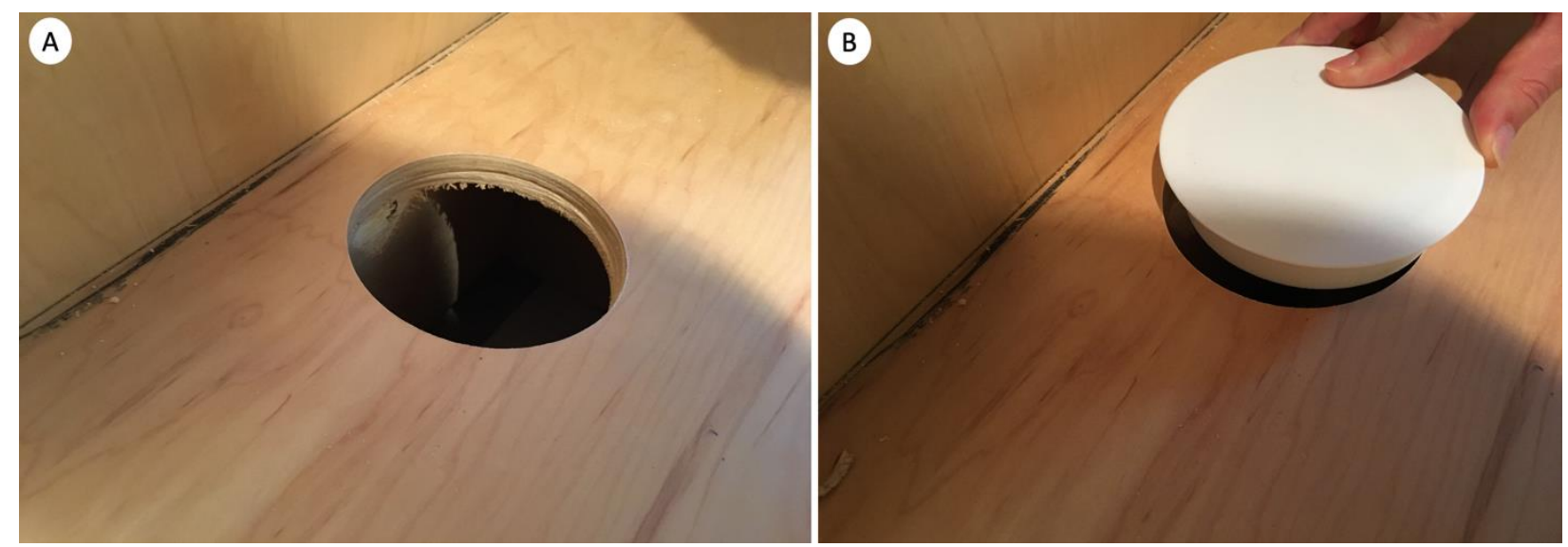

Figure 2. (A,B) Drilling of toe-kick voids in improved treatment homes (A). A fitted plastic cover designed to cover up the hole drilled at the bottom of cabinets after treatment for aesthetic reasons $(\mathbf{B})$. 


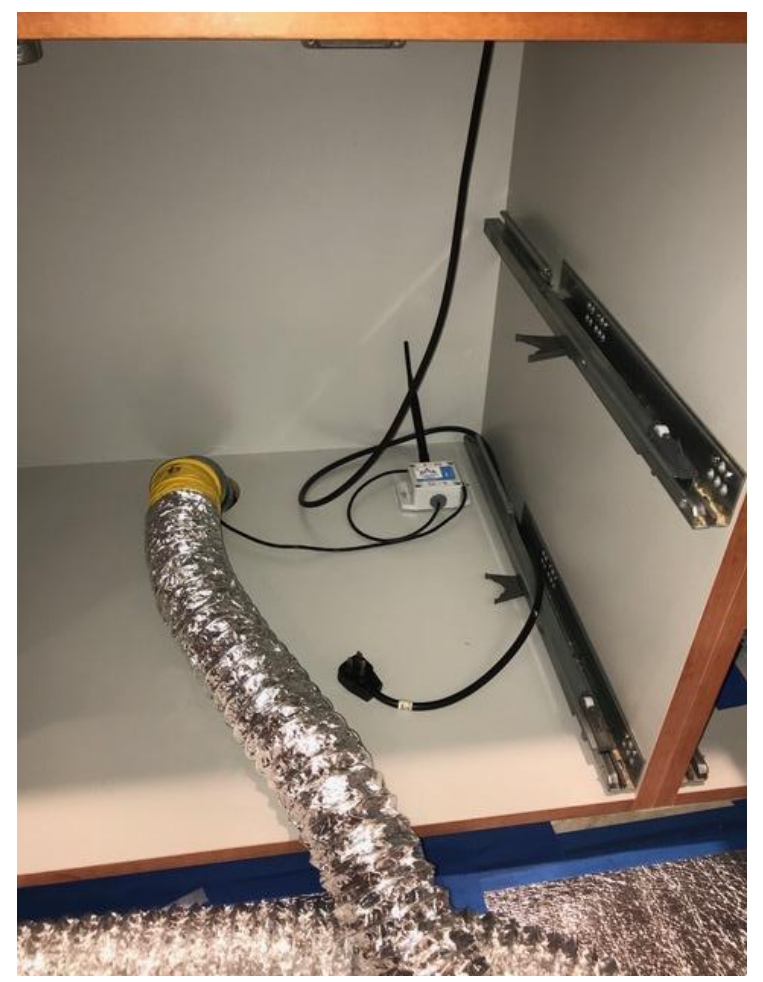

Figure 3. In each improved treatment home, probes (right) were placed in toe-kick voids for temperature monitoring and recording. Flexible duct pipes (left; with yellow cloth) were placed in toe-kick voids to direct heated air.
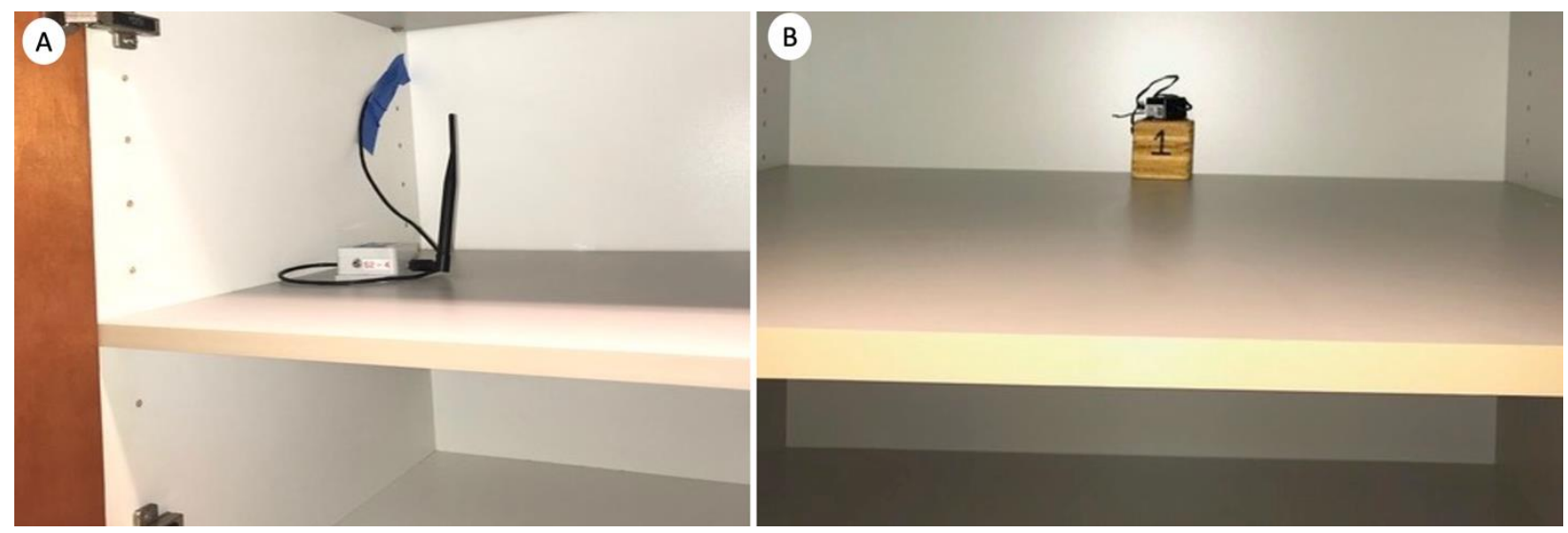

Figure 4. (A,B) Core temperature probes were installed by drilling to the center of the selected sidewall or side cabinet, placing the probe in the hole, and sealing the hole with duct tape (A). A custom-made wooden cube with an embedded temperature sensor was placed in a low-air circulation location for temperature recording $(\mathbf{B})$. 


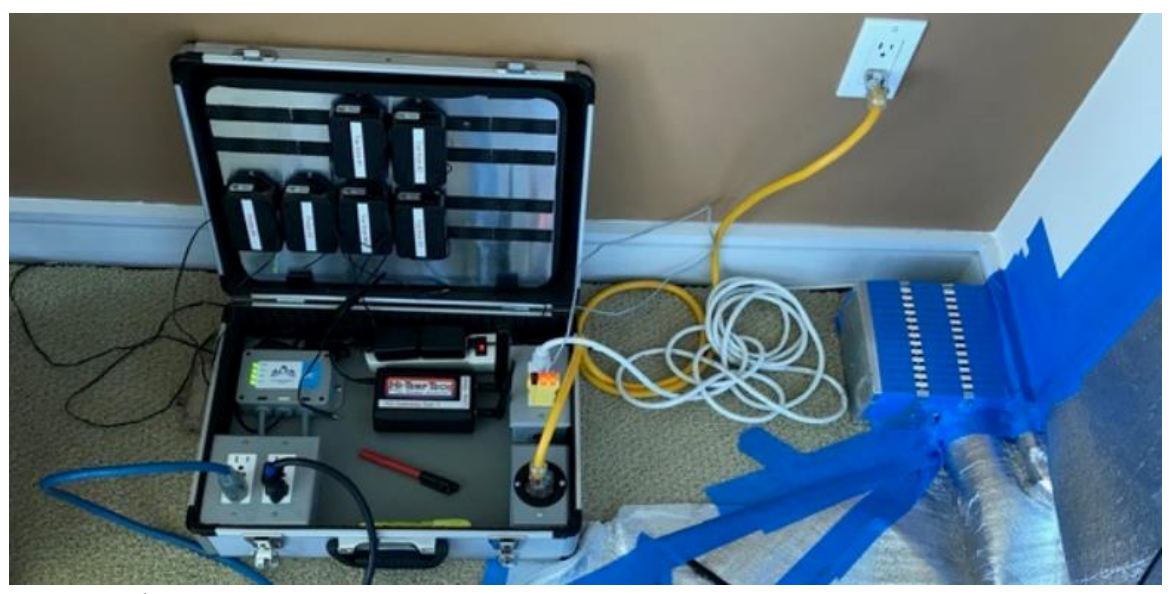

Figure 5. A temperature sensor control unit.

Three electric heaters with distribution manifolds were used to generate and direct heated air into the drilled four-inch-diameter holes in the base cabinet's toe-kick via threeinch-diameter flexible duct pipes (Figure 3) heated with air pressure and into the rest of the treatment zone. Nine multiple angle-adjustable high-velocity fans (XPOWER X-35AR axial blower fan, XPOWER, CA, USA), designed to handle high temperatures without blade distortion were positioned at multiple angles. The fans were aimed to drive heated air into corners and upper cabinets and to create air currents to avoid air cavities and dead spots (Figure 6a). Aluminized mylar blankets were placed from floor to ceiling as necessary to create contained, hermetically sealed heat zones (Figure 6b). This improves heating efficiency and reflects energy into the treatment areas. Instead of using propane heaters, relatively safer electric heaters (Elite Bed Bug Heater, K \& J Representatives LLC; AZ, USA) typically used for bedbug heat treatment were used together with fans. The heating protocols were adopted from current bedbug heat treatment protocols with some modification and improvement. In this study, once the target temperatures $\left(46{ }^{\circ} \mathrm{C}\right.$ or $\left.50{ }^{\circ} \mathrm{C}\right)$ were reached in all voids and treatment areas, the heaters were continued for 120 minutes and then shut off. In the current study, heat was applied for a total of 165 minutes. The target temperature was achieved and maintained during the last 120 minutes before shutting off the heaters. The data logger stopped recording the temperatures when the heaters were shut off (e.g., at the 165-minute time point).
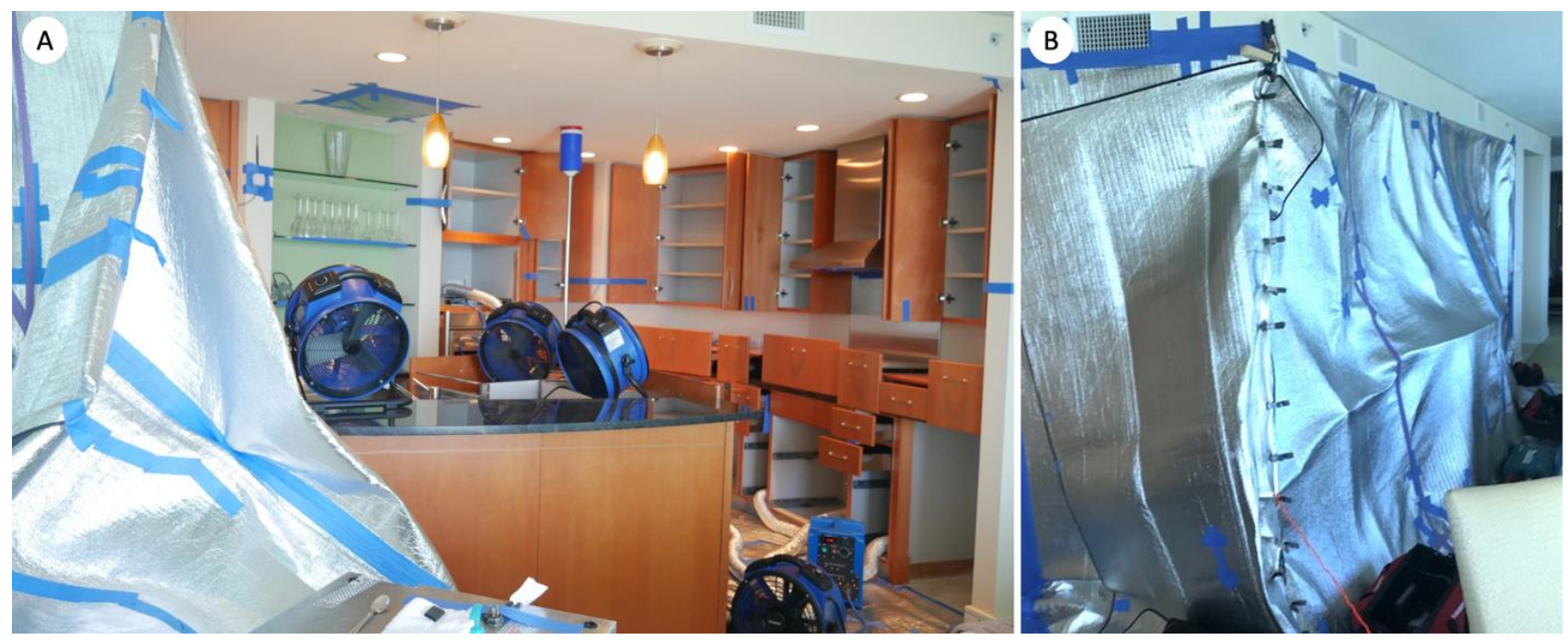

Figure 6. (A,B) Three heaters with flexible duct pipes, and nine fans in place for heated air circulation (A). A treatment zone covered with a thermal blanket to contain the heated air (B). 
For standard treatment (as control), a heat remediation company, HI-Temp Tech, LLC., conducted standard commercial high-temperature treatments at three residential homes with similar treatment zone sizes and termite infestation levels. Similar heat treatment durations, temperatures, and rates of temperature increase were recorded. However, these treatments were performed without drilling holes in the back of base cabinets, without duct pipes to direct heated air into toe-kick voids, and without placing temperature sensors inside the toe-kick voids. For standard heat treatments, the temperature sensors were placed inside the treatment zone, embedded inside cabinets or sidewalls, or placed in a wooden cube. The efficacy of the standard and improved heat treatments was compared at 6 months posttreatment by visual observation and using Termatrac. The callback rates at 6 months posttreatment due to residents noticing termite pellets were also compared.

\section{Results and Discussion}

During the inspections prior to heat treatment, a few C. brevis pseudergates were noticed in the standard and improved treatment homes via visual observation in the area of damaged wood, and the locations of termite infestations were further defined using a Termatrac T3i All Sensor. Termatrac is a device that uses low-energy microwaves to detect termite activity within most building materials, including wood. The Termatrac T3i All Sensor also has moisture and thermal sensors, which can detect moisture and measure surface temperatures. However, in this study, only the radar (i.e., low-energy microwave) sensor was used to detect termite activity.

In one of the improved treatment homes, the temperatures from eight wireless sensors from the online wireless portal (iMonnit, AZ, USA) showed that the temperatures recorded from the toe-kick areas (the top three lines in Figure 7) fluctuated but were still able to maintain an average above $50{ }^{\circ} \mathrm{C}$ throughout the treatment period. This fluctuation occurred because the enclosed toe-kick areas can accumulate a large amount of heat, causing the heater's internal programming to turn it on and off. The temperatures recorded from the ambient sensors (the fourth and fifth lines) and the core temperature probes inside the hole of the sidewall or side cabinet (the sixth and seventh lines) remained above $50^{\circ} \mathrm{C}$, whereas the temperatures recorded from the custom-made wooden cube with an embedded temperature sensor (the eighth line) remained steadily above $46^{\circ} \mathrm{C}$ (Figure 7). These temperatures are comparable to the original suggested treatment temperature of $49{ }^{\circ} \mathrm{C}$ [27], with the exception of the embedded temperature sensor in the layered-wood cube with temperatures above $46^{\circ} \mathrm{C}$ because the wooden cube was made thicker than the cabinet wood to represent worst case scenarios.

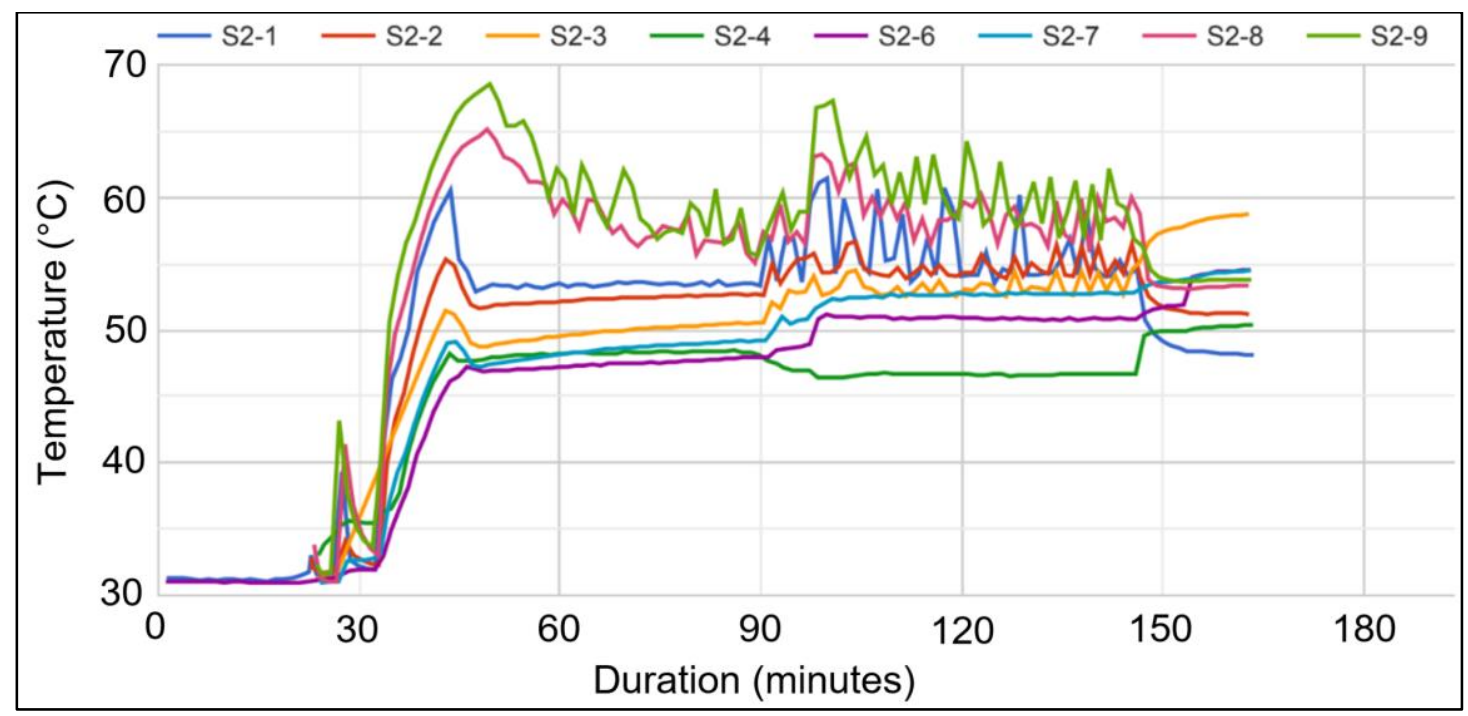


Figure 7. An overview of temperatures recorded by eight sensors/loggers (S2-1, S2-2, etc.) throughout the treatment period in one of the improved heat treatment homes via iMonnit portal's temperature data logger. Note the fluctuating high temperatures in the top three lines, representing the toe-kick probes.

The benefits of heat treatment are the ability to treat a whole structure without using pesticides and the relatively short period for which the structure must be vacated. For example, the occupants can return immediately once the heat treatment is completed. In contrast, during fumigation, the treated structure must be aerated and must remain vacated for at least 48 hours after treatment. Another advantage of heat treatment is the practicality of localized treatment, which allows it to be performed in portions of high-rise buildings, such as in apartments and condominium units, where fumigation, which must be performed in the whole structure, is not feasible. In certain circumstances, heat treatment provides greater flexibility to homeowners because they can choose whether the treatment will be applied to the entire unit or only to known areas of infestation (the kitchen, storage room, bedroom, etc.). Additionally, heat treatment has no residual toxicity, guaranteeing that the treated property is entirely safe for occupancy by humans and pets. It also poses a lower risk to applicators compared with chemical treatment [28]. No resistance or tolerance of insect pests to heat has been reported. When seeking regulatory approval, heat treatment requires less registration or authorization and thus reduces costs.

The major disadvantages of heat treatment include the difficulty or complexity of large infested structures and the longer time required to raise the internal core of the infested wood within such structures to lethal temperatures [24]. Increasing the heating time also results in an increase in the treatment cost, the time for which an area must be vacated, and the potential for heat damage to structural items. Cost is an important factor to consider because heat treatment is generally more expensive than chemical treatment. Potential damage may also occur to heat-sensitive items, such as plastics, electrical outlet covers, and cable wiring. Sensitive products, such as aerosol cans, fire extinguishers, and adhesives, should be removed before heat treatment. Roof and wall vents should be sealed. Cleaning and vacuuming are essential before heat treatment because debris, such as food residue, may act as heat insulators.

To ensure treatment efficacy, one of the main factors that we must consider is the presence of heat sink areas [24]. Using fans to circulate heated air helps improve the treatment but does not solve the problem completely. These structural heat sink areas are commonly found in the wood contacting concrete floors or foundations and tile floors. For example, cement is known to be a good heat conductor [29,30]. Hence, a longer heating duration is required to reach the target temperature in wood in contact with cement. Drywood termites tend to avoid high temperatures and may escape to these cooler zones in the wood and subsequently survive the heat treatment [31-33]. A recent laboratory study found that a temperature of approximately $49.6{ }^{\circ} \mathrm{C}$ for 2 hours is required to achieve $100 \%$ termite mortality with a heat sink effect [34]. However, temperature may increase slower in field applications than in laboratory studies [25]; thus, longer durations may be needed when conducting standard heat treatment in the field, as compared to the improved heat treatment in our study.

Maintenance of the lethal temperature for an adequate period is necessary to achieve treatment efficacy [17]. In the current study, relatively lower temperatures with a longer heating duration (a total of 165 minutes) were used. The heat sink zones were identified in the toe-kick voids where the wood members were in direct contact with the floor tiles. To mitigate heat sink effects in the improved treatment homes, the approach that we used in this study was to allow the heated air to be directed into toe-kick areas through duct pipes. Accordingly, four-inches holes were drilled in the back of all infested cabinets with a base with toe-kick voids. The three-inch flexible duct pipes were placed from the heaters into the drilled holes, creating sufficient heated air pressure into the void cavities, which is necessary to reach temperatures that allow the heat to transfer through construction 
materials and into the targeted areas (into all holes, gaps, and rear wall spaces). The oneinch diameter difference between the duct pipes and the holes allowed the back air pressure to escape from the holes. Meanwhile, the internal and ambient temperatures were monitored using probes or temperature sensors in several difficult-to-reach areas to ensure the temperature was sufficiently high to kill drywood termites. This ensured that more heat reached the toe-kick voids and that subsequently the target temperature was achieved. The temperature sensors consistently recorded temperatures above $50{ }^{\circ} \mathrm{C}$ in the toe-kick and other treatment areas and above $46^{\circ} \mathrm{C}$ in a wooden cube for 120 minutes. More fans were also used in relatively small treatment zones of approximately $3.0 \times 4.5 \mathrm{~m}^{2}$ than in other studies.

Taravati (2018) [26] has reported some limitations with Termatrac that may cause false-negative inspection results (i.e., detection of nontermite objects, movement by the person holding the device, reduced sensitivity with an increasing depth of the building materials, and termite inactivity during an inspection). In this study, in addition to inspection with Termatrac, the treatment zone was also thoroughly visually inspected for drywood termite frass (fecal pellets) and "kick-out holes."

All improved treatment homes had no termite activity at 6 months posttreatment according to Termatrac use (Figure 8) and careful visual inspections. Although drilling into the toe-kick voids required more labor and a higher cost, this step ensured complete elimination of the termite colonies and reduced the need for additional treatments in the future. Because only a few surviving termites were found be able to recover and develop neotenic reproductives [35], in the current study, a $33.33 \%$ callback (retreatment) rate occurred in homes that underwent the standard heat treatment, whereas a $0 \%$ callback rate occurred in homes that underwent the improved heat treatment after 6 months (Figure 9). Additionally, if the temperatures of all target sites including heat sink zones (e.g., toe-kick voids) can be monitored following the proposed protocols for improved treatment homes, either the temperature or the duration of heat treatment may possibly be reduced, shortening the period during which the structure must be vacated and reducing the treatment cost and the potential for heat damage to household items and furniture. Such careful heat treatments can provide a sustainable solution to drywood termite infestation.

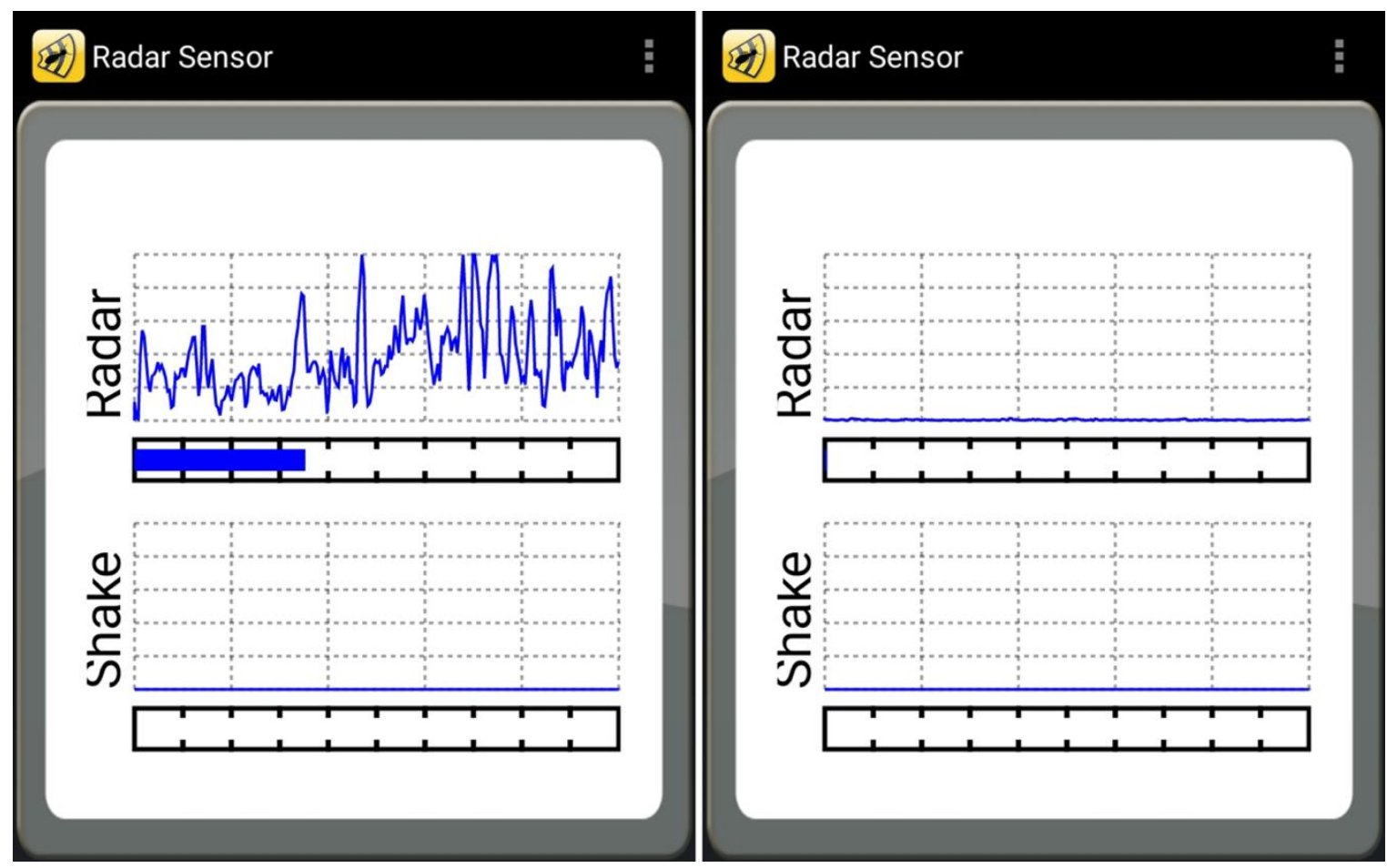

Figure 8. Termite activity present pretreatment (left) and absent at 6 months posttreatment (right) in one of the improved heat treatment homes. 


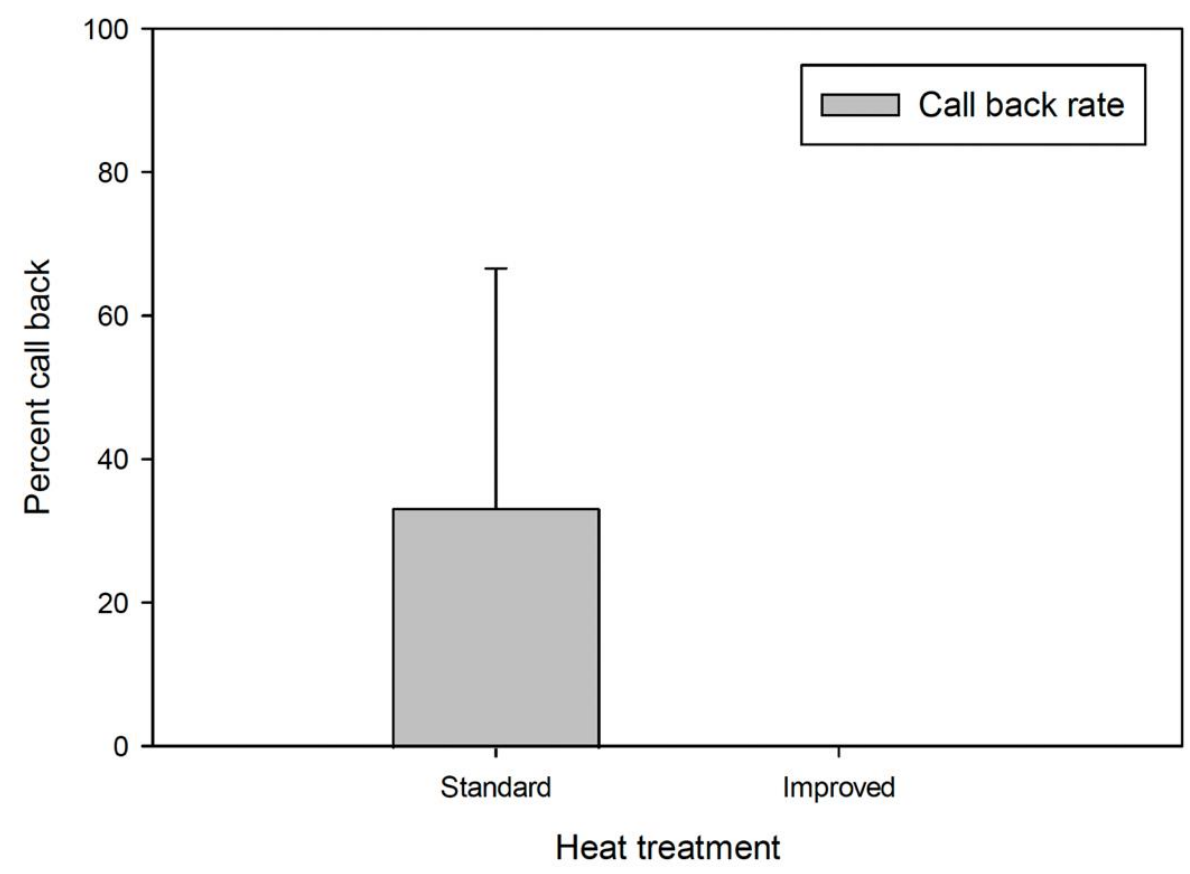

Figure 9. Callback (retreatment) percentages for standard and improved heat treatments.

\section{Conclusions}

Pest management professionals use several options for drywood termite treatment: local or spot treatment (i.e., pesticide liquids, foams, or dusts), fumigation, and heat treatment. Heat treatment is the only nonchemical option. Thus, heat treatment may be the most preferable option when residents have chemical and/or environmental concerns. Additionally, heat treatment is an attractive option for drywood termite-infested high-rise buildings, including condominium units, where fumigation is not feasible. This study was conducted in real-world residential properties using actual heating protocols that are currently used in typical heat treatments with our own improvements. The approaches used and protocols developed were efficacious against $C$. brevis in this study. The protocols were effective in addressing concerns regarding a standard or typical heat treatment on possible heat sink zones, and can help reduce incidences of retreatment or callbacks and possible heat damage to structural items due to the use of excessive temperature to overcome heat sink challenges.

Funding: Funding was partially provided by the USDA National Institute of Food and Agriculture, Integrated Extension Smith Lever POW and Integrated Hatch Project (accession number: 1022165), managed by the College of Tropical Agriculture and Human Resources, University of Hawaii at Manoa.

Institutional Review Board Statement: Not applicable.

Acknowledgments: We are grateful to J. Kenneth Grace and anonymous reviewers for their comments on the manuscript.

Conflicts of Interest: The authors declare no conflict of interest. The funders had no role in the design of the study; in the collection, analyses, or interpretation of data; in the writing of the manuscript, or in the decision to publish the results.

\section{References}


1. Scheffrahn, R.H.; Su, N.-Y.; Chase, J.A.; Mangold, J.R.; Grace, J.K.; Yates, J.R.III. First record of Cryptotermes cynocephalus Light (Isoptera: Kalotermitidae) and natural woodland infestations of C. brevis (Walker) on Oahu, Hawaiian Islands. Proc. Hawaii. Entomol. Soc., 2000, 34, 121-125.

2. Grace, J.K.; Woodrow, R.J.; Yates, J.R. Distribution and management of termites in Hawaii. Sociobiology, 2002, 40, 87-93.

3. Tong, R.L.; Grace, J.K.; Mason, M.; Krushelnycky, P.D.; Spafford, H.; Aihara-Sasaki, M. Termite species distribution and flight periods on Oahu, Hawaii. Insects, 2017, 8, 58.

4. Bess H.A. Termites of Hawai'i and the Oceanic Islands. In Biology of Termites; Krishna K., Weesner F.M. (eds.); Academic Press: New York, NY, USA, 1970; pp. 517-533.

5. Su N.-Y.; Scheffrahn, R.H. Economically important termites in the United States and their control. Sociobiology, 1990, 17, 77-94.

6. Scheffrahn R.H.; Su, N.-Y.; Busey, P. Laboratory and field evaluations of selected chemical treatments for control of drywood termites (Isoptera: Kalotermitidae), J. Econ. Entomol., 1997a, 90, 492-502.

7. Woodrow, R.J.; Grace, J.K.; Yates, J.R.III. Hawai'i's termites-An identification guide. In Household and Structural Pests; College of Tropical Agriculture and Human Resources: Honolulu, HI, USA, 1999; pp. 1-6.

8. Lewis, V.R.; Power, A.B.; Haverty, M.I. Laboratory evaluation of microwaves for control of the western drywood termite (Isoptera: Kalotermitidae). Forest Products Journal, 2000, 50, 79-87.

9. Su N.-Y; Scheffrahn R.H. Termites as pests of buildings. In Termites: Evolution, Sociality, Symbioses, Ecology, Abe T., Bignell D.E., Higashi M. (eds); Kluwer Academic Publishers: Boston, MA, USA, 2000; pp. 437-453.

10. Scheffrahn, R.H.; Křeček, J.; Ripa, R.; Luppichini, P. Endemic origin and vast anthropogenic dispersal of the West Indian drywood termite. Biol Invasions, 2009, 11, 787-799.

11. Evans T.A.; Forschler B.T.; Grace J.K. Biology of invasive termites: a worldwide review. Annu. Rev. Entomol., 2013, 58: 455-474.

12. Grace, J.K.; Cutten, G.M.; Scheffrahn R.H.; Kevan, D.K.McE. First infestation by Incisitermes minor of a Canadian building (Isoptera: Kalotermitidae). Sociobiology, 1991, 18, 299-304.

13. Rust, M.K; Su, N.-Y. Managing social insects of urban importance. Annu. Rev. Entomol., 2012, 57, $355-375$.

14. Hansen, J.D.; Johnson, J.A.; Winter, D.A. History and use of heat in pest control: a review. International Journal of Pest Management, 2011, 57, 267-289.

15. Fields, P.G; White, N.D.G. Alternatives to methyl bromide treatments for stored-product and quarantine insects. Annu. Rev. Entomol., 2002, 47, 331-359.

16. Kells, S.A.; Goblirsch, M.J. Temperature and time requirements for controlling bed bugs (Cimex lectularius) under commercial heat treatment conditions. Insects, 2011, 3, 412-422.

17. Lewis, V.R.; Forschler, B. Management of drywood termites: past practices, present situation and future prospects. In Urban insect pests: Sustainable management strategies; Dhang P. (ed.); CABI: Boston, MA, USA, 2014; pp. 130-153.

18. Suma P.; Chinnici, G.; La Pergola, A.; Russo, A.; Bella, S.; Pecorino, B.; Pappalardo, G. Assessing the technical effectiveness and economic feasibility of pest management through structural heat treatment: An entomological and economic analysis in four mills in Sicily (Italy). J. Econ. Entomol., 2019, 112, 957-962.

19. Ebeling, W. Wood-destroying insects and fungi. In Urban Entomology; Ebeling W. (ed.); University of California Press: Berkeley, CA, USA, 1975; pp. 143-167.

20. Woodrow, R.J.; Grace, J.K. Thermal tolerances of four termite species (Isoptera: Rhinotermitidae, Kalotermitidae). Sociobiology, 1998a, 32, 17-25.

21. Woodrow, R.J.; Grace, J.K. Laboratory evaluation of high temperatures to control Cryptotermes brevis (Isoptera: Kalotermitidae). J. Econ. Entomol., 1998b, 91, 905-909.

22. Scheffrahn, R.H.; Wheeler, G.S.; Su, N.-Y. Heat tolerance of structure-infesting drywood termites (Isoptera: Kalotermitidae) of Florida. Sociobiology, 1997b, 29, 237-245.

23. Lewis, V.R.; Haverty, M.I. 1996. Evaluation of six techniques for control of the western drywood termite (Isoptera: Kalotermitidae) in structures. J. Econ. Entomol., 1996, 89, 922-934.

24. Perry, D.T.; Choe, D.-H. Volatile essential oils can be used to improve the efficacy of heat treatments targeting the western drywood termite: evidence from simulated whole house heat treatment trials. J. Econ. Entomol., 2020a, 113, $2448-2457$.

25. Woodrow, R.J.; Grace, J.K. Field studies on the use of high temperatures to control Cryptotermes brevis (Isoptera: Kalotermitidae). Sociobiology, 1998c, 32, 27-50.

26. Taravati S. Evaluation of low-energy microwaves technology (Termatrac) for detecting western drywood termite in a simulated drywall system. J. Econ. Entomol., 2018, 111, 1323-1329.

27. Ebeling, W. Heat penetration of structural lumbers. IPM Practitioner, 1994, 11: 1-4.

28. Hammond, D. Heat treatment for insect control: developments and applications; Woodhead Publishing: Amsterdam, The Netherlands, 2015; pp. 1-120.

29. Rijke, A.M.; Rieger, M.R.; McLaughlin, R.E; McCoy, S. Porous acrylic cement. Journal of Biomedical Material Research, 1977, 11, 373-394.

30. Cui, H.; Liao, W.; Mi, X.; Lo, T.Y; Chen, D. Study on functional and mechanical properties of cement mortar with graphitemodified microencapsulated phase-change materials. Energy and Buildings, 2015, 105, 273-284.

31. Cabrera, B.J.; Rust, M.K. Behavioral responses to light and thermal gradients by the western drywood termite (Isoptera: Kalotermitidae). Environ. Entomol., 1996, 25, 436-445. 
32. Rust, M.K.; Reierson, D.A. Use of extreme temperatures in urban insect pest management. In Temperature sensitivity in insects and application in integrated pest management; Hallman G.J. and Denlinger D.L. (eds.); Westview Press: Boulder, CO, USA, 1998; pp. 179-200.

33. Cabrera, B.J.; Rust, M.K. Behavioral responses to heat in artificial galleries by the western drywood termite (Isoptera: Kalotermitidae). J. Agric. Urban Entomol., 2000, 17, 157-171.

34. Perry, D.T.; Choe, D.-H. Volatile essential oils can be used to improve the efficacy of heat treatments targeting the western drywood termite: evidence from a laboratory study. J. Econ. Entomol., 2020b, 113, 1373-1381.

35. Smith, M. Drywood termites: the experts answer some 'toughies'. Pest Control Tech, Fall 1995; pp. 34, 36-37, 40, 88. 